\title{
Transcranial magnetic stimulation: an alternative physical treatment in depression
}

\author{
Veronica O'Keane
}

Ir J Psych Med 2001; 18(3): 79-81

There are still a very limited number of physical therapies available for those suffering from depression, in spite of the rapid developments in pharmacological treatments. Electroconvulsive therapy (ECT) has the highest rate of response of any form of antidepressant therapy but is generally used only in those patients failing to respond to pharmacotherapy, except in specific and very limited situations where it is recommended as a primary therapy. Stereotactic subcaudate tractotomy (SST), the standard neurosurgical procedure employed in the UK for resistant depression, is only indicated when patients are suffering from severe, disabling depressive illness; and have failed to respond to all other treatments. Physical therapies are generally framed in this context, ie. as treatments for those suffering from intractable depression. Novel physical therapies will inevitably be compared to ECT as the standard physical treatment. The categorisation and comparison of new physical therapies with ECT may not be the appropriate way to proceed with evaluating the usefulness of such treatments. Transcranial magnetic stimulation (TMS) is a new technology for non-invasively stimulating the brain and may be an example of a treatment option that needs to be re-evaluated outside the context of resistant depression.

Transcranial magnetic stimulation (TMS): mechanism of action

TMS has been used as a tool for mapping brain function for over a decade. TMS involves placing an electromagnetic, insulated coil on the scalp and rapidly changing the currents in the coil so that magnetic fields are generated. These magnetic fields induce currents in neurones to a depth of about $2 \mathrm{~cm}$ from the coil surface; thus altering neuron function, probably by depolarisation. ${ }^{1}$ Different TMS frequencies result in different physiological effects: electrophysiological studies have shown that $1-\mathrm{Hz}$ rTMS of the primary motor area can produce a local decrease in excitability, whereas higher frequencies are associated with facilitation of motor cortical excitability. ${ }^{2,3}$ The effects obtained from TMS also depend on the interstimulus intervals (ISIs), as different neuronal circuits are recruited by differing ISIs. ${ }^{4}$ For example, intracortical inhibitory circuits are activated at ISIs of $1-4 \mathrm{~ms}$ and facilitatory circuits at ISIs of $8-12 \mathrm{~ms}^{5}$

\section{Effects of TMS}

Stimulation of selective cortical areas results in specific motor, sensory, cognitive and behavioural effects. ${ }^{6}$ Left temporal cortex stimulation can block speech production,

Veronica O'Keane, MB PhD MRCPsych FRCPI, Psychiatric Unit, Beaumont Hospital, Dublin 9, Ireland.

SUBMITTED: JUNE 19, 2001. ACCEPTED: AUGUST 21, 2001. and stimulation over the occipital cortex can induce visual disturbance. ${ }^{7,8}$ Stimulation over the left dorsolateral prefrontal cortex can inhibit working memory. ${ }^{9}$ Motor evoked potentials (MEP) in peripheral muscle results from stimulation of the motor cortex and this effect has been used to investigate hemispheric differences in motor cortical excitability in depression. ${ }^{10}$ This group compared MEPs rising from cortical stimulation on the right and left cerebral hemispheres in depressed and control groups and found that depressed patients showed lower excitability on the left and greater excitability on the right, compared to controls. This finding is in keeping with neuroimaging studies suggesting that depressed individuals have a relative reduction in left dorsolateral prefrontal activity. ${ }^{11,12}$ TMS has been used as an investigative tool in several neurological disorders including Parkinson's Disease, associated with reduced motor cortical inhibition at ISIs of less than $5 \mathrm{~ms}$, and cerebellar degeneration, associated with reduced cortical facilitation..$^{13,14}$

\section{TMS as an antidepressant therapy}

The use of TMS as a potential therapeutic tool derived from the observation that subjects experienced changes in affect during TMS over dominant frontal regions. ${ }^{1.5}$ Initial studies had equivocal results but with the development of rapid rate magnetic stimulators, using frequencies of up to $20 \mathrm{~Hz}$, and targeting the left dorsolateral prefrontal cortex (LDLPFC), more robust results were obtained. ${ }^{16,17,21)}$ TMS is administered following determination of the patient's individual motor threshold by evaluating the minimal stimulation over the motor cortex that will produce an MEP. This measurement is an index of cortical excitability and determines the stimulation for treatment (usually $80 \%-90 \%$ of motor threshold). A 'mapping' procedure identifies the LDLPFC: this site is located on the cortex by observing MEP or movement of the abductor pollicis brevis on the contralateral side. The LDLPFC is located $5 \mathrm{~cm}$ anterior to the optimal site of stimulation of this muscle.

\section{Clinical trials}

The first study to employ targeted TMS examined responses in six patients with treatment-resistant major depressive disorder (MDD). ${ }^{18}$ Two patients showed a slight, and two a marked, improvement. The results, although modest, were encouraging. Figiel et al ${ }^{19}$ examined the anti-depressant efficacy of TMS in 56 refractory patients in an open trial, as measured by a $60 \%$ reduction in HDRS with a final post-treatment score of 16 or less, and found a $42 \%$ response rate. Subsequent clinical trials of rTMS can broadly be divided into those in resistant depression, comparative trials with ECT and those in mildmoderate depression. Only placebo-controlled, or sham, studies will be reviewed. 
The first double-blind, randomised and controlled trial compared responses to sham and real TMS over the right and left DLPFC and vertex in 17 patients with drug-resistant, psychotic depression. ${ }^{20}$ Left DLPFC stimulation resulted in significant reduction in some depression scores compared to other treatments. Berman et $a^{21}$ assigned treatment-resistant patients to an active $(n=10)$ or sham ( $n=10)$ course of rTMS. A two week course of active rTMS resulted in statistically significant, but clinically modest reductions in depression scores, compared to sham. Padberg et al ${ }^{22}$ compared fast frequency, slow frequency and sham rTMS in a group of 18 patients with pharmacotherapy-resistant MDD. Although HDRS scores fell by $18 \%$, the authors concluded that rTMS did not show any clinically meaningful antidepressant efficacy. The most recent study compared real to sham rTMS in 40 patients with drug-resistant depression. ${ }^{23}$ Real, but not sham rTMS, was associated with a significant decrease in HDRS, but only 12 patients decreased more than $50 \%$. They concluded that rTMS targeting the LDLPFC was associated with antidepressant efficacy but that the size effect was small. One study found no difference between sham and real rTMS in 18 patients suffering from refractory depression. ${ }^{24}$ There were no apparent methodological differences between this study and others to explain the differing findings.

George et al ${ }^{17}$ compared sham to active LDLPFC TMS in out-patients with depression and found only a modest reduction in Hamilton Depression Rating Scores (HDRS). A subsequent study by this group ${ }^{25}$ examined the efficacy of TMS, without concurrent antidepressant use, in 30 outpatients with MDD. Compared to sham, active TMS produced significantly greater reductions in HDRS but response rates were only $45 \%$ in the active group. A study from Russia compared real to sham rTMS, in combination with psychotherapy, in 29 patients with 'neurotic depression'. ${ }^{24}$ HDRS fell from a mean of 22.9 to 8.6 in the treatment group and was significantly lower than the drop in scores in the sham group.

Studies comparing ECT to rTMS have employed differing designs. One protocol, employed in two studies, compares ECT to rTMS in a head-to-head design. One of these studies found superiority for ECT in some measures but found no overall differences between the two groups, as measured by rates of remission and drop in HDRS. ${ }^{27}$ Another study randomly allocated 40 patients to either ECT or rTMS and found ECT to be superior in delusional depression but there were no differences in outcome between the two treatment groups in MDD without psychosis. ${ }^{28}$ Another design examined the efficacy of alternating ECT with rTMS treatments vs ECT alone. ${ }^{29}$ Combination therapy was as effective as ECT alone and not associated with as many subjective side-effects.

\section{Safety and tolerability of rTMS}

TMS is considered a safe treatment if used within guidelines for maximum safe combination of stimulus frequency, intensity, ISI and duration. ${ }^{16}$ A study of 22 patients who had brain volumetric analysis performed using MRI, prior to and following rTMS, found that there were no observed structural changes as a result of treatment. ${ }^{30}$ The most frequent unwanted side effects are mild scalp discomfort due to muscular contraction, and noise. The use of ear plugs is recommended for this reason. Auditory threshold was examined in 18 subjects who received up to six weeks exposure to rMRS and two patients showed mild high frequency hearing loss after several weeks. ${ }^{31}$

Neuropsychological function has been tested formally in several studies of rTMS and has been found: to improve globally following treatment, probably as a result of practice effects (as the improvement was also found in the sham group); ${ }^{31}$ to selectively improve memory function only; ${ }^{32}$ and to have no adverse effects on neuropsychological performance. ${ }^{33}$

\section{Mechanism of action of rTMS in depression}

The pathophysiology of depression may include hypoactivity in the left prefrontal cortex, as demonstrated by neuroimaging studies. ${ }^{11}$ One mechanism of action of rTMS in depression may be to increase activity in the DLPFC brain region. Several studies have tested this hypothesis, using diverse research strategies. Catafau et $a^{l^{34}}$ examined regional cerebral blood flow ( $\mathrm{rCBF}$ ) in seven patients with MDD before and following treatment with rTMS. They found that treatment induced a significant increase in rCBF in the left prefrontal cortex but no relationship could be established between increases in rCBF and clinical response. Another single photon emission computed tomography (SPECT) study examined brain blood flow changes in 22 depressed adults following a two week course of standard rTMS therapy. ${ }^{35}$ At medication-free baseline blood flow in the bilateral medial temporal lobes, left prefrontal cortex and caudate declined with increasing depression severity. This negative correlation disappeared following treatment. Responders showed increased inferior frontal lobe activity compared to non-responders prior to treatment and this difference increased further following treatment.

A neuroimaging study using positon emission tomography (PET) has examined rCBF in 10 patients using high and low frequency rTMS. ${ }^{36}$ High frequency rTMS resulted in increased $\mathrm{rCBF}$ in bilateral frontal, limbic and paralimbic regions, whereas low frequency rTMS produced more circumscribed decreases. Taken together, these results suggest that rTMS affects prefrontal and paralimbic activity, but the relationship between this effect and treatment response is not clear.

Rat studies have inevitably examined changes in brain serotonin (5HT) function consequent to rTMS. One study found that $\mathrm{TTMS}$ reduced the sensitivity of 5HT1A autoreceptors: a finding also demonstrated following antidepressant treatments. ${ }^{37}$ Another study has found that rTMS in 'high-anxiety' rats attenuates behavioural and neuroendocrine (cortisol) stress responses. ${ }^{38}$ Comparable studies in humans are lacking.

\section{Conclusions}

Neuropsychopharmacological treatments for depression continue to advance but the area of physical somatic interventions has lagged behind. TMS therapy undoubtedly has antidepressant actions, as evidenced by the above studies. It has the advantage of not requiring anaesthesia, not inducing seizure and has no apparent cognitive side effects. Preliminary data from rTMS and PET studies reveal differential effects of rTMS frequency on regional cerebral neural activity in depressed patients. That rTMS may be more efficacious in severe depression, compared to $\mathrm{mild} /$ moderate depression, may be because rTMS is targeting a specific brain dysfunction present only in more 'biologically' defined depressions. These findings are also 


\section{You made her life more complete...}

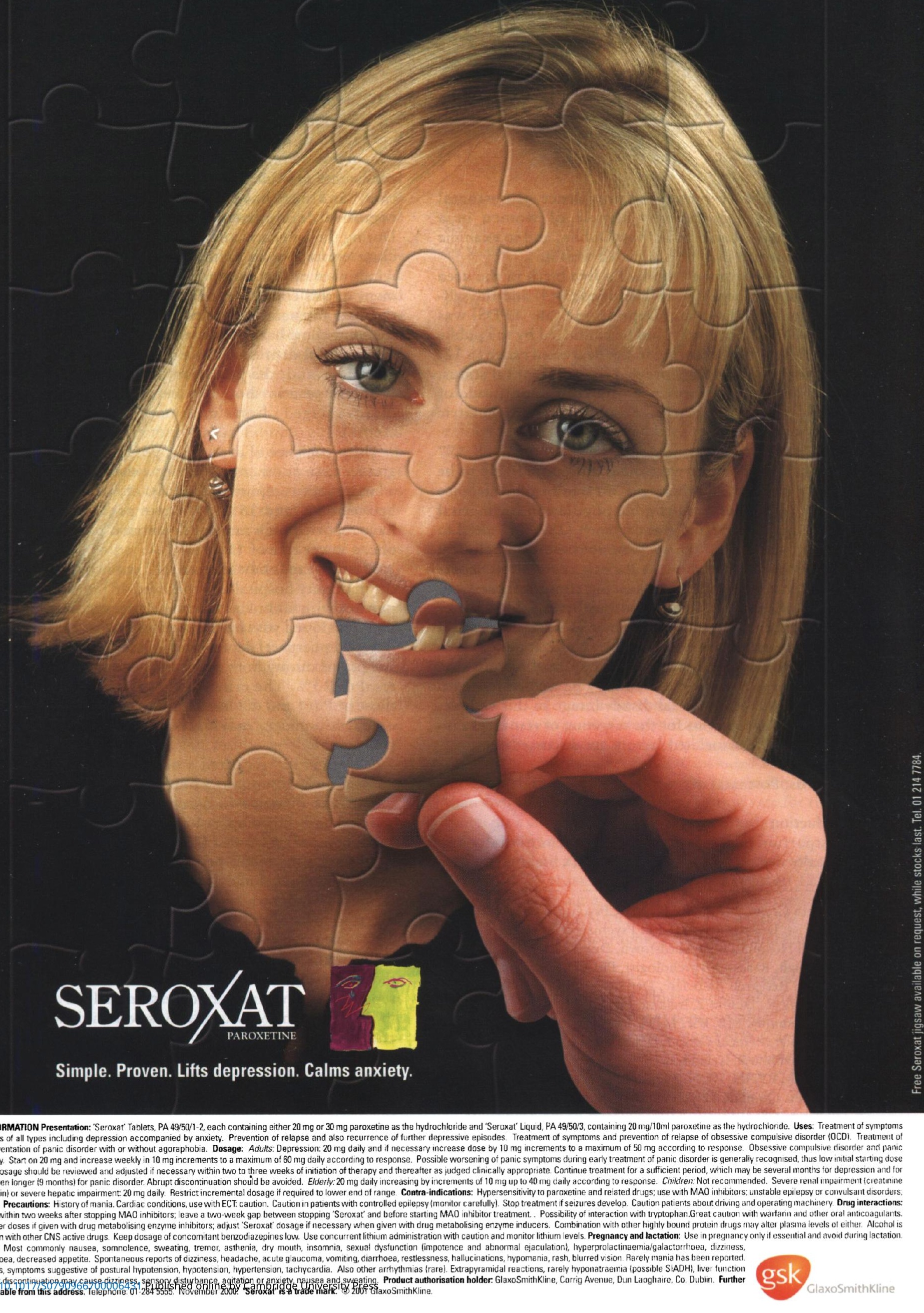


exciting because it lends further weight to the evidence implicating left prefrontal cortex dysfunction in depression. Repetitive TMS is not effective in psychotic depression, in contrast to ECT, and suggests that another brain area may be involved in processing the psychotic symptoms of depression.

TMS is not only a valuable treatment option but informs us about the nature of different depressive syndromes. Clearly, depression is a heterogenous disorder, defined in terms of clinical syndromes rather than brain pathophysiology. That rTMS has not yet found a clinical niche may result from the limitations of our definitions of depression, rather than of the therapy itself. Therapies such as rTMS may enable us to move towards more biologically intelligent definitions of depressive syndromes.

\section{References}

1. Rudiak, Marg E. Finding the depth of magnetic brain stimulation: a reevaluation. Electroencephalography and Clinical Neuropsysiology 1994; 93 : 358-71.

2. Chen R, Classen J, Gerloff C et al. Depression of motor cortex excitability by low-frequency transcranial magnetic stimulation. Neurology 1997; 48: 1398-403.

3. Wasserman EM, Wedegaertner RF, Ziemann U, George MS, Chen R. Crossed reduction of human motor excitability $1-\mathrm{Hz}$ transcranial magnetic stimulation. Neurosci Lett 1998; 10: 141-4.

4. Pascual-Leone A, tormos JM, Keenan PJ et al. Study and modulation of human cortical excitability with transcranial magnetic stimulation. J Clin neurophysiol 1998; 15: 333-43.

5. Ziemann U, Rothwell JC, Ridding MC. Interaction between intracortical inhibition and facilitation in human motor cortex. J of Psyiol 1996; 496: 87381.

6. Wassermann EM. Repetitive transcranial magnetic stimulation: an introduction and overview. CNS Spectrums 1991; 2: 21-5.

7. Pascual-Leone A, Gates JR, Dhuna A. Induction of speech arrest and counting errors with rapid-rate transcranial magnetic stimulation. Neurology $1991 ; 41: 697-702$.

8. Amassian E, Cracco RQ, Maccabee PJ et al. Suppression of visual perception by magnetic coil stimulation of human occipital cortex. Electroencephalography Clin Neurophysiol 1989; 4: 458-62.

9. Pascual-Leone A, Hallett $M$. Induction of errors in a delayed response task by repetitive transcranial magnetic stimulation of the dorsolateral prefrontal cortex. Neuroreport 1994; 5:2517-20.

10. Maeda F, Keenan, JP, Pascual-Leone A. Interhemispheric asymmetry of motor cortical excitability in major depression as measured by transcranial magnetic stimulation. B J Psych 2000; 177: 169-73.

11. Baxter LR, 'Schwartz JM, Phelps ME et al. Reduction of prefrontal cortex glucose metabolism common to three types of depression. Arch Gen Psychiatry $1989 ; 46: 243-50$.

12. George M.S, Lisanby SH, Sackeim HA. Transcranial magnetic stimulation. Applications in neuropsychiatry. Arch Gen Psychiatry 1999; 56: 300-11.

13. Berardelli A, Rona S, Inghilleri $M$ et al. Cortical inhibition in Parkinson's disease. A study with paired magnetic stimulation. Brain 1997; 119: 71-7. 14. Liepert J, Wessel K, Schwenkreis P et al. Reduced intracortical facilitation in patients with cerebellar degeneration. Acta Neurolog Scand, 1998; 98: 318-23 15. Bickford RG, Guidi M, Fortesque P et al. Magnetic stimulation of human peripheral nerve and brain: response enhancement by combined magnetoelectrical technique. Neurosurg 1987; 20:110-6.

16. Pascual-Leone A, Wassermann E.M. Repetitive transcranial magnetic stimulation: application and safety considerations. In Advances in Occupational Medicine and Rehabilitation (Nilsson J, Panizza M, Grandori F. eds). Pavia, Italy: Fondazione Salvatore Maugeri Edizione. 1996.

17. George MS, Wassermann EM, Kimbrell, TA et al. Mood improvement following daily left prefrontal repetitive transcranial magnetic stimulation in patients with depression: A placebo-controlled crossover trial. Am Journal of Psych 1997; 154: 1752-6.

18. George, MS, Wassermann EM, Williams WA et al. Daily repetitive transcranial magnetic stimulation ( $\mathrm{TTMS}$ ) improves mood in depression. NeuroRpor $1995 ; 6: 1853-6$.

19. Figiel GS, Epstein C, McDonald WM et al. The use of rapid rate transcranial magnetic stimulation (rTMS) in refractory depressed patients. J Neuropsych Clin Neurosci 1998; 10: 20-5.

20. Pascual-Leone A, Rubio B, Pallardo $F$ et al. Rapid rate transcranial magnetic stimulation of the left dorsolateral prefrontal cortex in drug-resistant depression. Lancet 1996; 348: 233-7.

21. Berman RM, Narasimhan M, Sanacora $G$ et al. A randomised clinical trial of repetitive transcranial magnetic stimulation in the treatment of major depression. Biol Psychiatr 2000; 47: 332-7.

22. Padberg F, Zwanzger $\mathrm{P}$, Thoma $\mathrm{H}$ et al. Repetitive transcranial magnetic stimulation (rTMS) in pharmacotherapy-refratory major depression: comparative study of fast, slow and sham rTMS. Psychiatry Res 1999; 88: 163 71.

23. Garcia-Toro M. Mayol A, Arnillas $\mathrm{H}$ et al. Modest adjunctive benefit with transcranial magnetic stimulation in medication-resistant depression. J Affect Disord 2001; 64: 271-5.

24. Loo C, Mitchell, P, Sachdev P et al. Double-blind controlled investigation of transcranial magnetic stimulation for the treatment of resistant major depression. Am J. Psychiatry 1999; 156: 946-8.

25. George MS, Nahas Z, Molloy M et al. A controlled trial of daily left prefrontal cortex TMS for treating depression. Biol Psychiatry 2000; 48: 95961.

26. Stikhina NI, Lyskov EB, Lomarev MP et al. Transcranial magnetic stimulation in neurotic depression. Zh Nevrol Psikhiatr Im S S Korsakova 1999; 99: $26-9$

27. Pridmore S. Bruno R, Turnier-Shea $Y$ et al. Comparison of unlimited numbers of rapid transcranial magnetic stimulation (rTMS) and ECT treatment sessions in major depressive episode. Int J Neuropsychopharmacol 2000; 2 : 129-34.

28. Grunhaus L, Dannon PN, Schreiber S et al. Repetitive transcranial magnetic stimulation is as effective as electroconvulsive therapy in the treatment of nondelusional major depressive disorder: an open study. Biol Psychiatry 2000 47: 314-24.

29. Pridmore S. Substitution of rapid transcranial magnetic stimulation treatments for electroconvulsive therapy treatments in a course of electroconvulsive therapy. Depress Anxiety 2000; 12: 118-23.

30. Nahas Z, DeBrux C, Chandler V et al. Lack of significant changes on magnetic resonance imaging scans before and after 2 weeks of daily left prefrontal repetitive transcranial magnetic stimulation for depression. J. ECT $2000 ; 16: 380-90$.

31. Loo C, Sachdev P, Elsayed H et al. Effects of a 2-4 week course of repetitive transcranial magnetic stimulation (rTMS) on neuropsychologic functioning, electroencephalogram, and auditory threshold in depressed patients. Bio Pychiatry 2001; 49:615-23.

32. Little, J.T., Kimbrell, T.A., Wassermann, E.M. et al. Cognitive effects of 1and 20-hertz repetitive transcranial magnetic stimulation in depression: preliminary report. Neuropsych Neuropsychol Behav Neurol 2000:13: 119124.

33. Triggs WJ, McCoy KJ, Greer R et al. Effects of left frontal transcranial magnetic stimulation on depressed mood, cognition, and corticomoto threshold. Biol Psychiatry $1999 ; 45: 1440-6$.

34. Catafau AM, Perez V, Gironell A et al. SPECT mapping of cerebral activity changes induced by repetitive transcranial magnetic stimulation in depressed patients. A pilot study. Psychiatry Res 2001; 106: 151-60.

35. Teneback CC, Nahas Z, Speer AM et al. Changes in prefrontal cortex and paralimbic activity in depression following two weeks of daily left frontal TMS J Neuropsychiatry Clin Neurosci 1999; 11: 426-35.

36. Speer AM, Kimbrell TA, Wassermannm EM et al. Opposite effects of high and low frequency rTMS in regional brain activity in depressed patients. Biol Psychiatry 2000; 48: 1133-11.

37. Gur E, Lerer B, Dremencov E et al. Chronic repetitive transcranial magnetic stimulation induces subsensitivity of presynaptic serotonergic autoreceptor activity in rat brain. Neuroreport 2000; $11: 2925-9$

38. Keck ME, Welt T, Post A et al. Neuroendocrine and behavioural effects of repetitive transcranial magnetic stimulation in a psychological animal model are suggestive of antidepressant-like effects. Neuropsychopharmacology 2001; 24: $337-49$.

\section{The Irish Journal of Psychological Medicine welcomes all correspondence by email}

\section{Email us at:}

\section{psychological@medmedia.ie}

